\title{
A magneto-optical probe for the investigation of multiphase Fe oxide nanosystems
}

Giulio Campo ${ }^{*}$, Francesco Pineider ${ }^{2,1}$, Valentina Bonanni ${ }^{1}$, Martin Albino ${ }^{1}$, Andrea Caneschi ${ }^{1}$,

César de Julián Fernández ${ }^{3}$, Claudia Innocenti ${ }^{1}$, Claudio Sangregorio*4

1 INSTM and La.M.M, Department of Chemistry U. Schiff, Università di Firenze, Via della Lastruccia 3, 50019 Sesto Fiorentino (FI), ITALY

2 CNR-ISTM, Via Marzolo 1, 35131 Padova, ITALY

3 CNR-I.M.E.M, Parco Area delle Scienze 37/A, 43124 Parma, ITALY

4 CNR-ICCOM and INSTM, Via Madonna del Piano 10, 50019 Sesto Fiorentino (FI), ITALY

\begin{abstract}
In iron oxide nanoparticles the transformation of the metastable magnetite phase to maghemite, through the oxidation of iron, often represents a major drawback for the correct interpretation of their physical properties and their effective use in several applicative areas. To solve this issue we propose an innovative method for identifying the chemical composition of complex ferrite nanostructures based on magneto-optical (MO) spectroscopy. Spherical iron oxide nanoparticles with increasing size are investigated by magnetic circular dichroism (MCD) in the nUV-Vis-nIR
\end{abstract}


range $(350-1000 \mathrm{~nm})$. Particular attention is dedicated to the time evolution of the MO response, which is ascribable to the oxidation process of the nanomaterial. The measured MCD spectra show a complex lineshape due to the overlap of several MO transitions. A deconvolution analysis of MCD hysteresis loops allows the interpretation of this complex response as the combination of two distinct contributions, originating from magnetite and maghemite domains in the nanoparticles. We consider this method potentially competitive in terms of simplicity and accuracy with respect to conventional techniques for the discrimination of the two magnetic phases in nanostructured materials.

KEYWORDS: Magneto-optics, Magnetic Circular Dichroism, Iron oxide, Ferrite, Magnetic Nanoparticle

\section{INTRODUCTION}

Magnetic iron oxide nanostructures are very promising for a wide range of technological applications, such as electronics, data storage, catalysis and biomedicine. ${ }^{1-4}$ Among iron oxides, magnetite $\left(\mathrm{Fe}_{3} \mathrm{O}_{4}\right)$ is undoubtedly the best known, and its magnetic properties have been extensively studied. ${ }^{5}$ Due to the high Curie temperature of $850 \mathrm{~K}$ and strong spin polarization at room temperature, bulk magnetite finds application in giant magneto-electronic and spin-valve devices. ${ }^{6}$ When reducing size towards the nanoscale, the increased surface/volume ratio yields unusual chemical and physical behavior, which can modify and, when properly tailored, also enhance the magnetic properties. ${ }^{7}$ A precise knowledge of the crystalline structure thus becomes of fundamental importance for controlling the properties of these nanoconfined systems. In fact, from a chemical point of view, magnetite represents a metastable state as the possible oxidation 
of $\mathrm{Fe}^{2+}$ ions can convert it to maghemite, $\gamma-\mathrm{Fe}_{2} \mathrm{O}_{3}$. In bulk the composition of magnetite can be reasonably considered homogeneous, with the exception of the surface layers formed by air oxidation, whose contribution can in general be neglected. On the other hand, the large surface/volume ratio makes NPs very sensitive to the surrounding environment, and a larger oxidation of the $\mathrm{Fe}^{2+}$ ions might take place. ${ }^{8-10}$

Magnetite crystallizes in the inverse spinel structure, the formula being $\left(\mathrm{Fe}^{3+}\right)\left[\mathrm{Fe}^{3+} \mathrm{Fe}^{2+}\right] \mathrm{O}_{4}$, where round parentheses represent tetrahedral sites and square brackets the octahedral ones. Such structure originates a ferrimagnetic order, composed of two anti-parallel aligned magnetic sublattices, each one defined by ions occupying the same kind of cavities, with a net spin magnetic moment $m=4 \mu_{B}$ per formula unit. Maghemite, which also has a spinel structure, is not prone to oxidation due to the presence of $\mathrm{Fe}^{3+}$ ions only, distributed between tetrahedral and octahedral sites. Vacancies are present in the octahedral sites, so that the actual structural formula is $\left(\mathrm{Fe}^{3+}\right)\left[\square_{1 / 3} \mathrm{Fe}_{5 / 3}^{3+}\right] \mathrm{O}_{4}$ where $\square$ denotes vacancies. Maghemite maintains ferrimagnetic ordering with a net spin magnetic moment for a pure ferrimagnetic Néel model, reduced to $3.3 \mu_{B}$. Despite the structural similarity, $\mathrm{Fe}_{3} \mathrm{O}_{4}$ and $\gamma-\mathrm{Fe}_{2} \mathrm{O}_{3}$ exhibit rather different properties, e.g., mass density, electrical conductivity, magnetization density. ${ }^{5}$ The contribution of the two phases thus needs to be accurately determined for technological applications. For instance, the amount of $\mathrm{Fe}_{3} \mathrm{O}_{4}$ in NPs for biomedical application must be carefully evaluated due to the potential toxicity of $\mathrm{Fe}^{2+}$ ions. ${ }^{11}$

The problem of identifying the chemical composition of a spinel ferrite at the nanoscale is exacerbated by the fact that most of standard characterization techniques, such as X-ray diffraction (XRD), do not allow an accurate discrimination between magnetite and maghemite phases when the crystalline domains are smaller than $20 \mathrm{~nm}$. Standard magnetometric techniques 
are equally impaired in the identification of the two phases, since the values of most of the magnetic parameters that are usually considered for the discrimination of magnetic phases, i.e. saturation magnetization, magnetization dynamics, temperature dependence of susceptibility, can vary wildly in the case of nanostructuration. There is a single feature that is sharply different between the magnetic behavior of magnetite and maghemite: the presence at ca. $120 \mathrm{~K}$ of the Verwey transition exclusively in the former. ${ }^{12}$ However, in the case of nanostructured iron oxide, the observation of such transition has been reported to be evanescent, in particular for particle size ranges under $50 \mathrm{~nm},{ }^{6}$ thus making it an unreliable probe for NPs. Mössbauer and Raman spectroscopy, as well as x-ray absorption near edge spectroscopy (XANES), ${ }^{13}$ are the most suitable techniques for discriminating among iron oxide phases; on the other hand, the first two need a considerable amount of material which is not always available, whereas the latter requires large scale facilities.

In this landscape, the analysis of the magneto-optical (MO) behavior can represent an alternative and complementary experimental route for obtaining information on the composition of mixed iron oxide nanostructures particularly when only small amount of material is available. 14-16 Magneto-optics in fact probes the magnetic response of the sample via its optical transitions: in the simplest scenario, the optical transitions of different materials can be well separated spectrally, so that by measuring the MO hysteresis loop at different photon energies one can resolve the magnetic behavior of the individual constituents of the sample, in a way that is reminiscent of the element selectivity in synchrotron-based X-ray magnetic circular dichroism. In most cases, however, valence band transitions are broad and often overlap extensively, so this simple approach cannot be used. 
The method we describe here uses both the spectroscopic and magnetometric aspects of magnetic circular dichroism (MCD), ${ }^{17}$ a particular type of $\mathrm{MO}$ technique that measures the difference $\Delta \mathrm{A}=\mathrm{A}_{-}-\mathrm{A}_{+}$in the optical absorption of the left- and right-circularly polarized (LCP, RCP) states of the light crossing a magnetized medium; we propose this method as an efficient way to deconvolve spectrally overlapping magneto-optical terms of magnetite and maghemite using benchtop, cryogen-free instrumentation and a rather simple and fast experimental procedure. To achieve this, we investigated the MCD response at room temperature of a set of three iron oxide NP systems with increasing average diameter of 5, 6.5 and $10 \mathrm{~nm}$ (denoted as S-5, S-6.5 and S-10, respectively), as a function of the incident light wavelength and of the applied magnetic field. The MO properties of the systems have been also monitored for a period ranging from some days to 24 months of air exposition after the NPs have been synthesized and dispersed in a polymer matrix, thus giving us a picture of the progressive evolution of the samples from magnetite to maghemite. Our study turned out to be useful also for gathering information about the time stability of the chemical composition of oxide NPs in a polymer matrix.

Finally, we studied two standard systems of pure $\mathrm{Fe}_{3} \mathrm{O}_{4}$ and $\gamma-\mathrm{Fe}_{2} \mathrm{O}_{3}$ NPs, and three samples made up of a mixture of these standards. To further prove the validity of our method, we employed it to recover the single magnetic contributions in the mixed samples.

\section{EXPERIMENTAL SECTION}

Synthesis. The investigated magnetic samples are made of spherical iron oxide NPs of different size, prepared by colloidal chemistry. Samples S-5, S-5b, S-6.5 and S-10 were prepared by following the method described by Sun et al. ${ }^{18}$, S-10 was prepared by iterative decomposition 
using S- 6.5 as the starting seeds. The standard magnetite S-46 sample has been synthesized by following a modified version of the method described by Park et al. ${ }^{19}$. All the samples were prepared using commercially available reagents which were used as received.

Synthesis of $5 \mathrm{~nm}$ iron oxide NPS (S-5, S-5b). Fe(acac) $)_{3}(0.706 \mathrm{~g}, 2 \mathrm{mmol}), 1,2$-hexadecanediol (2.584 g, $10 \mathrm{mmol})$, oleic acid (1.695 g, $6 \mathrm{mmol})$, oleylamine (1.605 g, $6 \mathrm{mmol})$ and phenyl ether $(20 \mathrm{ml})$ were mixed and magnetically stirred under a flow of nitrogen in a $100 \mathrm{ml}$ three-neck round-bottom flask. The mixture was heated to $200{ }^{\circ} \mathrm{C}$ for $30 \mathrm{~min}$ and then, under a blanket of nitrogen, heated to reflux $\left(265^{\circ} \mathrm{C}\right)$ for another $30 \mathrm{~min}$. After cooling, product was recovered by precipitation with ethanol and subsequent centrifugation. The obtained product was then washed several times with ethanol and re-dispersed in hexane.

Synthesis of $6.5 \mathrm{~nm}$ iron oxide NPs (S-6.5). The sample was prepared with the same procedure as the $5 \mathrm{~nm}$ sample, using benzyl ether instead of phenyl ether, with the difference that the reagents mixture was heated to $200{ }^{\circ} \mathrm{C}$ for $2 \mathrm{~h}$ and then, under a blanket of nitrogen, heated to reflux $\left(\sim 300^{\circ} \mathrm{C}\right)$ for $1 \mathrm{~h}$.

Synthesis of $10 \mathrm{~nm}$ iron oxide NPs (S-10) via S-6.5 seeds. Fe(acac) $3(0.706 \mathrm{~g}, 2 \mathrm{mmol}), 1,2-$ hexadecanediol (2.584 g, $10 \mathrm{mmol})$, oleic acid $(0.565 \mathrm{~g}, 2 \mathrm{mmol})$, oleylamine (0.535 g, $2 \mathrm{mmol})$ and benzyl ether $(20 \mathrm{ml})$ were mixed and magnetically stirred under a flow of nitrogen in a 100 $\mathrm{ml}$ three-neck round-bottom flask. A $0.084 \mathrm{~g}$ of S-6.5 dispersed in hexane $(4 \mathrm{ml})$ was added. The mixture was first heated to $100{ }^{\circ} \mathrm{C}$ for 30 min to remove hexane, then to $200{ }^{\circ} \mathrm{C}$ for $1 \mathrm{~h}$. Under a blanket of nitrogen, the mixture was further heated to reflux $\left(\sim 300^{\circ} \mathrm{C}\right)$ for $30 \mathrm{~min}$. Sample recovery and purification were carried out as above. 
Synthesis of the precursor, Fe-oleate complex. $\mathrm{FeCl}_{3} \cdot 6 \mathrm{H}_{2} \mathrm{O}(2.22 \mathrm{~g}, 8.22 \mathrm{mmol})$ and Na-oleate (7.54 g, $24.75 \mathrm{mmol})$ were dissolved in a mixture containing $16 \mathrm{ml}$ of ethanol, $12 \mathrm{ml}$ of deionized water and $30 \mathrm{ml}$ of hexane and heated to reflux at $70{ }^{\circ} \mathrm{C}$ for $4 \mathrm{~h}$.

After removal of the aqueous phase, the red-brownish organic layer containing the iron oleate was washed three times with $6 \mathrm{ml}$ of deionized water and then dried under vacuum at $70{ }^{\circ} \mathrm{C}$ for $24 \mathrm{~h}$ obtaining a red-brownish waxy solid.

Synthesis of $46 \mathrm{~nm} \mathrm{Fe}{ }_{3} \mathrm{O}_{4} \mathrm{NPs}$. Fe(oleate) $)_{3}(2.31 \mathrm{~g}, 2.58 \mathrm{mmol})$, oleic acid $(2.157 \mathrm{~g}, 7.64 \mathrm{mmol})$ and 1-octadecene $(20 \mathrm{ml})$ were mixed and magnetically stirred under a flow of nitrogen in a 100 $\mathrm{ml}$ three-neck round-bottom flask. The reaction mixture was heated to reflux $\left(320^{\circ} \mathrm{C}\right)$ with a heating rate of $5{ }^{\circ} \mathrm{C} / \mathrm{min}$ under a blanket of nitrogen and was kept at that temperature for $4 \mathrm{~h}$. The black-brown solution was cooled to room temperature and a mixture of toluene/hexane/isopropanol ( $3 \mathrm{ml} / 10 \mathrm{ml} / 25 \mathrm{ml})$ was added to precipitate the NPs. The NPs were collected with a permanent magnet, washed three times by a mixture of hexane, isopropanol and ethanol and re-dispersed in toluene.

Preparation of MIX samples. The MIX dispersions were prepared by mixing liquid dispersions of S-5b and S-46 NPs with final concentrations of, respectively, $0.34 \mathrm{mg} / \mathrm{ml}$ and $0.55 \mathrm{mg} / \mathrm{ml}$ for MIX-A, $0.53 \mathrm{mg} / \mathrm{ml}$ and $0.43 \mathrm{mg} / \mathrm{ml}$ for MIX-B, and $0.74 \mathrm{mg} / \mathrm{ml}$ and $0.30 \mathrm{mg} / \mathrm{ml}$ for MIX-C.

Characterization. Average diameter and size distribution of NPs were determined by transmission electron microscopy (TEM), performed by a CM12 PHILIPS Transmission Electron Microscope operating at $100 \mathrm{kV}$ accelerating voltage. Powder X-Ray Diffraction (XRD) measurements were carried out using a Bruker D8 Advance diffractometer equipped with a $\mathrm{CuK} \alpha$ radiation and operating in $\theta-2 \theta$ Bragg Brentano geometry at $40 \mathrm{kV}$ and $40 \mathrm{~mA}$. 
Magnetometric measurements were performed on powder samples using a SQUID magnetometer (Quantum Design MPMS XL-5), operating in the 1.8-350 K temperature range and with an applied field up to $5 \mathrm{~T}$.

MCD characterization at room temperature was carried out using a home-built setup realized following Ref ${ }^{20}$, capable to perform magneto-optical measurements at room temperature for wavelengths ranging from $350 \mathrm{~nm}$ to $1000 \mathrm{~nm}$, and using magnetic fields up to $1.3 \mathrm{~T}$. The light source is a $200 \mathrm{~W} \mathrm{Hg-Xe} \mathrm{arc} \mathrm{lamp.} \mathrm{To} \mathrm{maximize} \mathrm{the} \mathrm{signal-to-noise} \mathrm{ratio} \mathrm{we} \mathrm{performed} \mathrm{our}$ measurements using a polarization modulation technique; the light coming from the monochromator, linearly polarized by using a Glan-Thompson polarizer, is modulated between the LCP and RCP polarizations at a frequency of $50 \mathrm{kHz}$, by passing through a photo elastic modulator (PEM). From the detector, the oscillating part of the electric signal is recovered with a phase sensitive technique by a lock-in amplifier locked to the frequency of the PEM. In our setup the sample is placed in a static magnetic field, parallel to the propagation direction of the light. The magnitude of dichroism signal $\Delta \mathrm{A}$ has been calibrated through a standard technique using a $\mathrm{Fe}(\mathrm{CN}) 6^{3+}$ solution for reference. MCD measurements were performed on NPs dispersed in polymethil-methacrylate (PMMA) matrices and cast into thin, optically addressable films. The preparation and storage of the films were carried out under atmospheric conditions. This form was preferred to a solvent dispersion in order to prevent the orientation of the NPs under a magnetic field application which can modify the correct probing of the magnetic properties. Moreover, due to the comparatively high magnetic moment of transition metal oxides, NPs migration under magnetic field gradients present at the edge of the cuvette would lead to instabilities in particle concentration in the probed area. 


\section{RESULTS AND DISCUSSION}
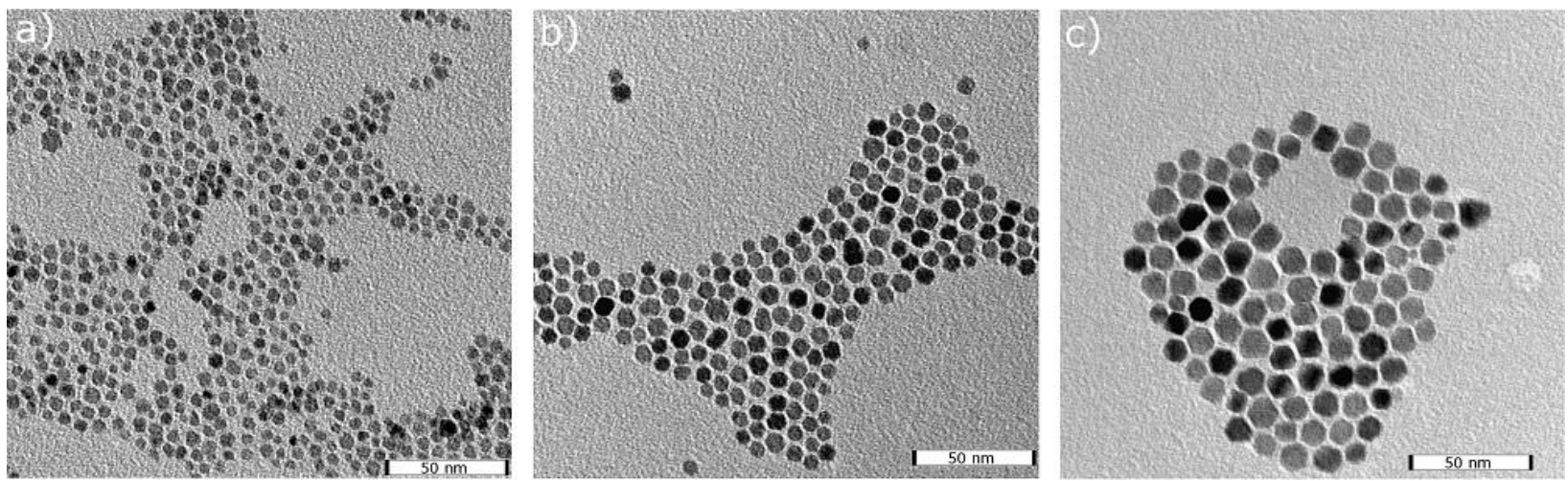

Figure 1. Low magnification TEM images of the synthesized NPs with average size: a) $5.0 \mathrm{~nm}$ (S-5); b) $6.5 \mathrm{~nm}(\mathrm{~S}-6.5)$; c) $10.1 \mathrm{~nm}(\mathrm{~S}-10)$.

All the freshly synthesized samples exhibited the expected black color, typical of magnetite. ${ }^{5}$ The TEM analysis of the samples (Figure 1) shows NPs with spherical shape and homogeneous size, with average diameters of $5.0 \pm 0.8 \mathrm{~nm}(\mathrm{~S}-5), 6.5 \pm 0.7 \mathrm{~nm}(\mathrm{~S}-6.5)$, and $10.1 \pm 1.1 \mathrm{~nm}(\mathrm{~S}-$ 10). The XRD patterns reveal that all the samples have the cubic spinel structure characteristic of ferrite (a representative example is given in Supporting Information, Figure S1). As expected, the broadening of the peaks due to the reduced size of the NPs does not allow the unambiguous discrimination between $\mathrm{Fe}_{3} \mathrm{O}_{4}$ and $\gamma-\mathrm{Fe}_{2} \mathrm{O}_{3}$.

Zero-Field-Cooled (ZFC) and Field-Cooled (FC) magnetization curves measured on freshly prepared powder samples are shown in Figure 2. A superparamagnetic behavior is observed at room temperature; blocking temperatures, $\mathrm{T}_{\mathrm{B}}$, coherently increase with the NPs average size from 32 to $115 \mathrm{~K}$. Moreover, S-6.5 and S-10 NPs display a steep increase of the magnetization at $110 \mathrm{~K}$, which is related to the Verwey transition. ${ }^{12}$ Interestingly, although it occurs at the same 
temperature in the two systems, the Verwey transition loses intensity by reducing the particle size and it is not observed at all in the smallest sample S-5.

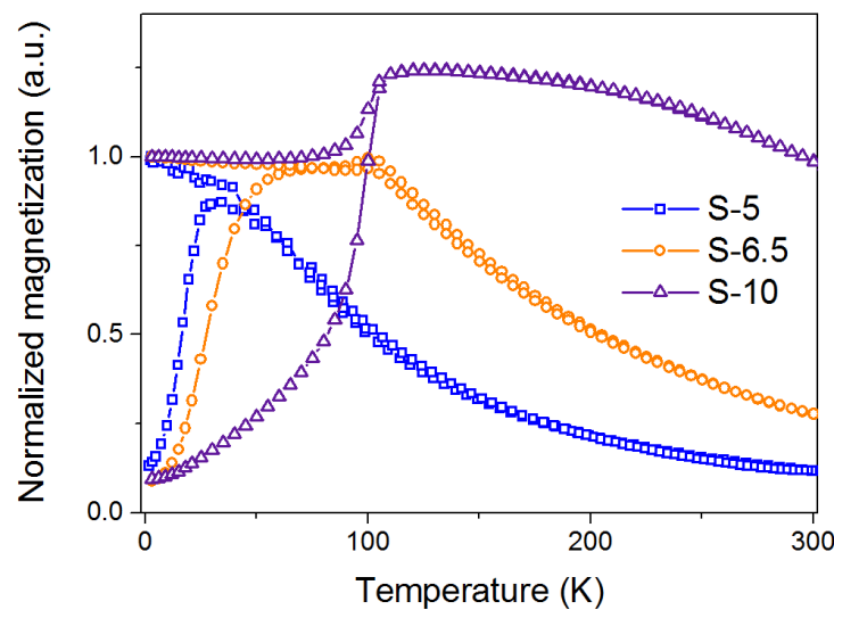

Figure 2. Temperature dependence of ZFC-FC magnetization curves, for freshly prepared iron oxide NPs with different sizes.

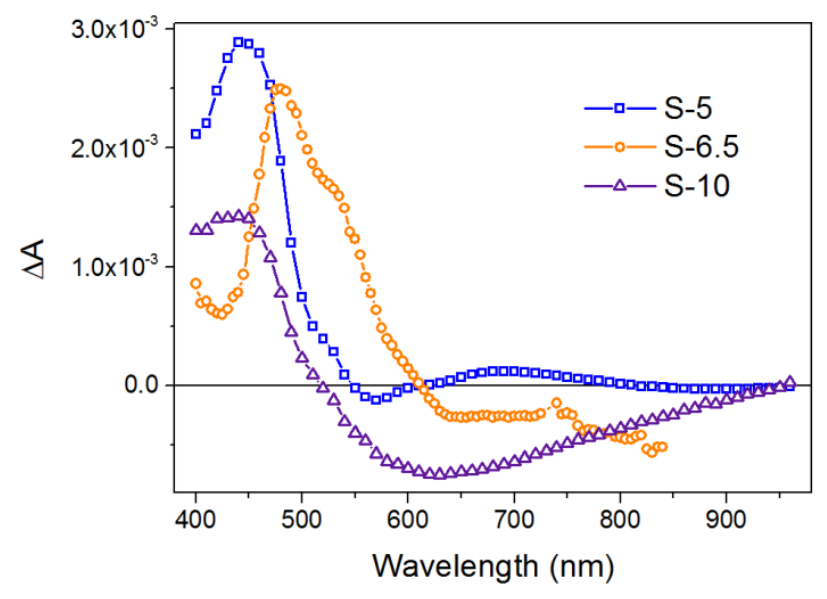

Figure 3. MCD spectra of iron oxide NPs with different sizes measured 3 months after the synthesis. 
The MCD spectra of the three samples were recorded 3 months after the synthesis on thin polymeric films obtained by dispersing the NPs in PMMA. The MO behavior of the three samples, displayed in Figure 3, is significantly different. Even though our nanostructured samples exhibit multiple overlapping MO transitions too broad to be resolved, three main spectral features can be recognized: the first one is an intense positive peak centered at $440 \mathrm{~nm}$ (shifted of ca. $50 \mathrm{~nm}$ for S-6.5), while the second one is a weaker positive peak centered at 530 $\mathrm{nm}$. The third feature, at longer wavelengths, is represented by a broad peak, which is positive for S-5 and negative for S-6.5 and S-10.

The interpretation of the MCD spectra of iron oxides is rather complicated due to the complexity of their electronic structure. Indeed, the $3 \mathrm{~d}$ electronic levels of $\mathrm{Fe}$ ions are spread into multiplets by the electron-electron Coulomb repulsion and further split by the crystal field. Such complexity has led to a number of different interpretations in the assignment of the MO transitions. An exhaustive analysis of the $\mathrm{MO}$ features of $\mathrm{Fe}_{3} \mathrm{O}_{4}$ and substituted ferrites was presented by Fontijn et al. ${ }^{21}$ In MCD measurements, the possible detectable transitions are limited by spin and parity rules; accordingly, two kinds of electronic transitions dominate the optical features of spinel ferrites: Crystal Field (CF) and Charge Transfer transitions. The CF transitions take place between $3 \mathrm{~d}$ states of a single metal ion, which are separated in energy by the ligand field generated by the surrounding $\mathrm{O}$ anions; Intervalence Charge Transfer (IVCT) and Intersublattice Charge Transfer (ISCT) transitions involve different cations. ${ }^{22}$ Because of selection rules, the CF transitions are in principle forbidden in ferrites when involving Fe cations placed in octahedral coordination and are weak for Fe ions in tetrahedral sites, although structural distortion can strongly increase the transition probability. The optical properties of $\mathrm{Fe}_{3} \mathrm{O}_{4}$ are dominated by several IVCT and ISCT transitions involving $\mathrm{Fe}^{2+}$ and $\mathrm{Fe}^{3+}$ ions which 
are, respectively, strongly- and weakly-dependent on the $\mathrm{Fe}^{2+}$ presence. ${ }^{23,24} \mathrm{In}$ the fully oxidized spinel $\gamma-\mathrm{Fe}_{2} \mathrm{O}_{3}$, the most intense features appear in the 400-515 nm (2.4-3.1 eV) range, usually corresponding to $\mathrm{CT}$ transitions involving $\mathrm{Fe}^{3+}$ cations; ${ }^{25}$ a possible alternative process consists in a simultaneous double excitation of $\mathrm{CF}$ transitions between two $\mathrm{Fe}^{3+}$ centers in two neighboring coordination sites coupled antiferromagnetically $\left({ }^{6} \mathrm{~A}_{1}+{ }^{6} \mathrm{~A}_{1} \rightarrow{ }^{4} \mathrm{~T}_{1}+{ }^{4} \mathrm{~T}_{1}\right) .{ }^{22}$

In order to get more insight on the interpretation of the data, the MCD measurements were repeated for all the samples after 12 and 24 months of air exposition. The comparison with the spectra obtained after 3 months is shown in Figure $4 \mathrm{a}$ and $4 \mathrm{~b}$ for S-5 and S-10, respectively. The S-5 spectrum does not exhibit a significant shape evolution, indicating that the system has a stable chemical composition. On the other hand, the MCD spectrum of the S-10 NPs displays a marked modification over time, particularly pronounced for longer wavelengths, suggesting that some reactive components were present. These results are compatible with the assumption that the largest particles (S-10) initially include mixed valence Fe ions, i.e. magnetite, which progressively converts to maghemite over time, whereas sample $\mathrm{S}-5$ prevalently contains $\mathrm{Fe}^{3+}$ ions at the time of the first measurement, which are stable toward further oxidation. The different reactivity is ascribed to the larger surface exposed by the smallest NPs. 


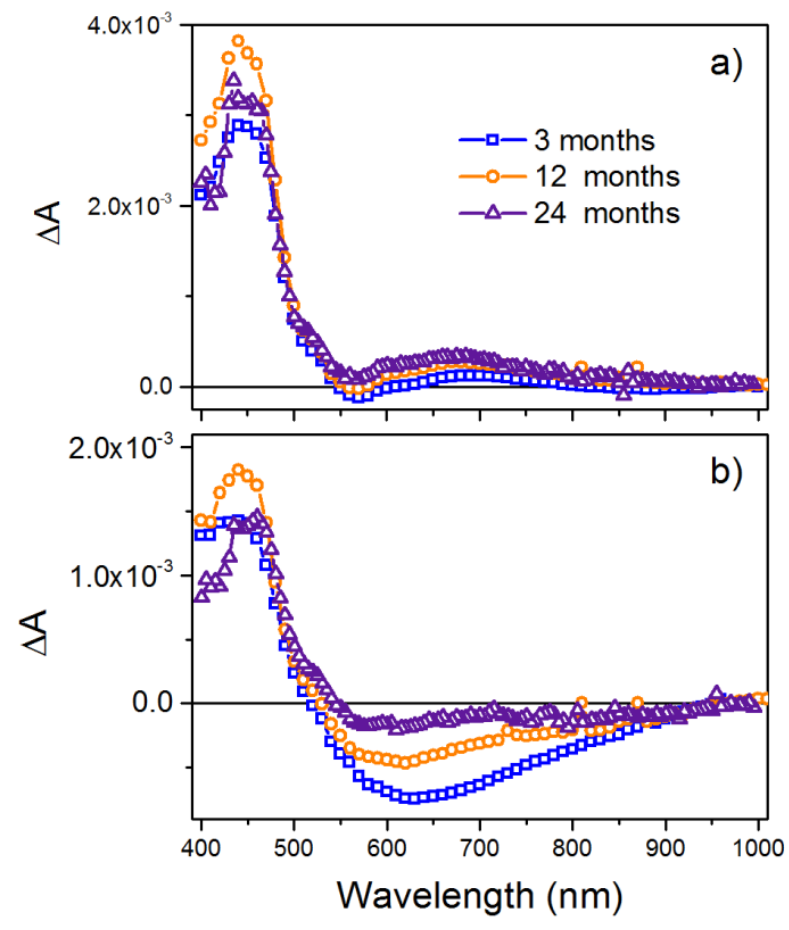

Figure 4. Time evolution of the MCD spectra of (a) S-5 and (b) S-10 NPs.

To support such hypothesis, the time evolution of the MCD spectra was monitored on a shorter time scale on a freshly prepared sample, S-5b, synthesized under the same conditions as S-5 and with similar size and size distribution (Fig. S2a). The MCD characterization was performed on S5b after the following time steps: 1) freshly prepared; 2) after 8 days of storage in $\mathrm{N}_{2}$ atmosphere; 3) after 8 days of storage in air; 4) after 18 months under air exposition. As shown in Figure 5, the sample remains unaltered after $\mathrm{N}_{2}$ exposition, whereas a clear evolution takes place already after 8 days of air exposition. Interestingly, the MCD spectrum evolution exhibits a pronounced reduction of the signal above $500 \mathrm{~nm}$, as already observed for $\mathrm{S}-10$. The evolution of $\mathrm{S}-5 \mathrm{~b}$ is consistent with the hypothesis that very small NPs are extremely prone to atmospheric oxidation. 


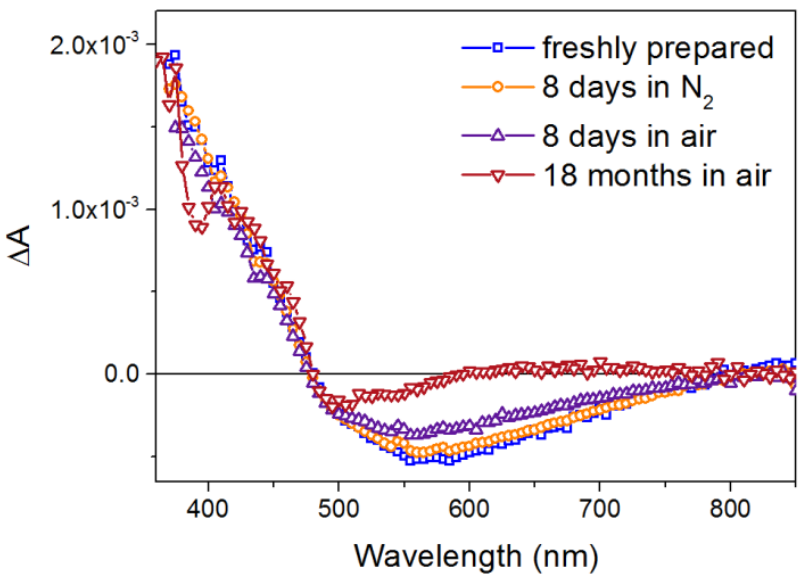

Figure 5. MCD spectra showing the time evolution of S-5b.

A more robust description of the iron oxides phases in our samples can be provided by a deep analysis of the wavelength-dependence of the MCD hysteresis loop. Indeed, this is a powerful tool to inspect the magnetic field response of different electronic transitions throughout the spectrum. While standard magnetometric techniques measure the average response of all magnetic phases over the whole sample, MCD hysteresis loops are strongly affected by the optical and MO constants of the individual magnetic components. The variation of the incident wavelength can thus correspond to changing the measurement sensitivity to each component of the system. ${ }^{26}$

The shape of the loops of our systems changes dramatically with the wavelength, varying from a regular superparamagnetic behavior to a more complex one. In Figure 6, the measured loops for S-5 are shown as a representative example. A strong variation of the shape of the hysteresis loops due to the presence of multiple magnetic components was already reported in the literature for $\mathrm{NiFe} / \mathrm{Au} / \mathrm{Co} / \mathrm{Au}$ thin films. ${ }^{27}$ Following a similar approach, we attempted to model the magnetic dependence of our samples by considering the sum of two magnetic 
contributions, which can be ascribed to the presence of different iron oxide phases, i.e. magnetite and maghemite. In particular, we devised a fitting method that we propose as an effective tool for the deconvolution of the different magnetic components.

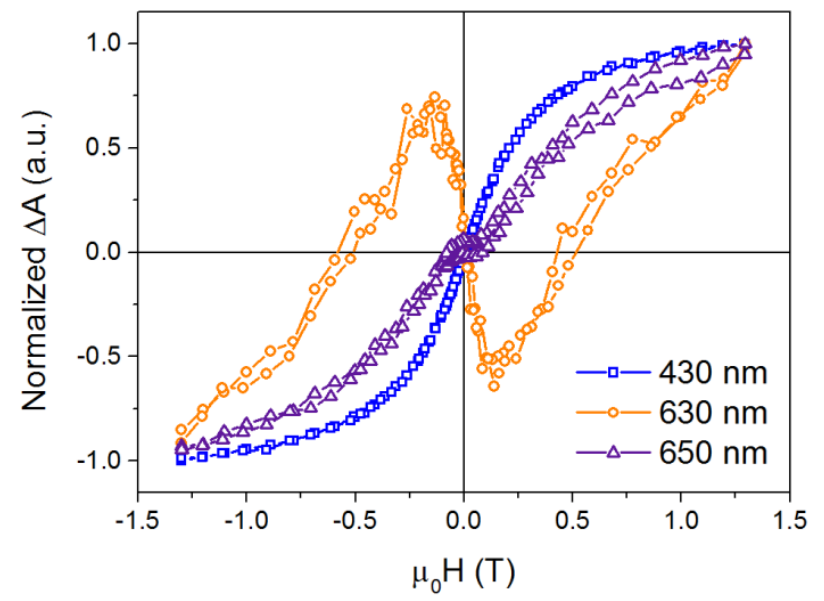

Figure 6. Normalized MCD loops recorded at different wavelengths for S-5 sample.

In a first approximation, both contributions have been considered to arise from superparamagnetic components; hence the fitting function has the form:

$$
\operatorname{MCD}(H)=\sum_{i=1,2} A_{s p_{i}} \mathcal{L}\left(C_{i} H\right)+A_{\text {lin }} H
$$

where $\mathcal{L}$ is the Langevin function $\mathcal{L}(\mathrm{x})=\operatorname{coth}(\mathrm{x})-1 / \mathrm{x}$ and $C_{i}$ is related to the saturation magnetization and the volume of $i$-th superparamagnetic component. $A_{s p_{i}}$ is the MCD amplitude of the $i$-th superparamagnetic contribution at a given wavelength. $A_{\text {lin }}$ is the amplitude of a linear term taking into account for the contributions arising from support, matrices and optical elements; they can have either diamagnetic or paramagnetic behavior but, for low field values, can be gathered in a single term, linear with the magnetic field H. For each sample, the fitting procedure was performed as follows: in the first step, $C_{1}\left(C_{2}\right)$ was fitted setting to zero the 
amplitude $A_{2}\left(A_{1}\right)$ and choosing MCD loops measured in a spectral region where the shape remains essentially unaltered with the wavelength, thus suggesting that only one of the two phases dominates the local MO response. Then the complementary $C_{2}\left(C_{1}\right)$ parameter was determined by fitting to eq. $\left({ }^{* *}\right)$ the MCD hysteresis loops where the presence of two magnetic components was recognizable. The spectral regions fulfilling the requirement for the first step were at around $400 \mathrm{~nm}$ and at wavelengths larger than $650 \mathrm{~nm}$. In the following we will generically denote the component showing the maximum MO signal at $\lambda \approx 400 \mathrm{~nm}$ and $\lambda>650$ $\mathrm{nm}$ as phase 1 and phase 2, respectively. By using this procedure, for each sample a couple of the parameters $C_{1}$ and $C_{2}$ was univocally determined. In the second step, for each sample the MCD loops recorded over the whole wavelength range were fitted with eq. $\left.{ }^{* *}\right)$ by keeping constant the corresponding $\left(C_{1}, C_{2}\right)$ couple. The fit yielded a couple of $A_{s p_{i}}$ parameters for each wavelength, which represent the intensity of the MCD response arising from each magnetic phase. This kind of approach provides useful information about the contributions involved as, in principle, it is possible to reconstruct and isolate the MCD spectral lineshape of the $i$-th component by plotting $A_{s p_{i}}$ as a function of the wavelength. 


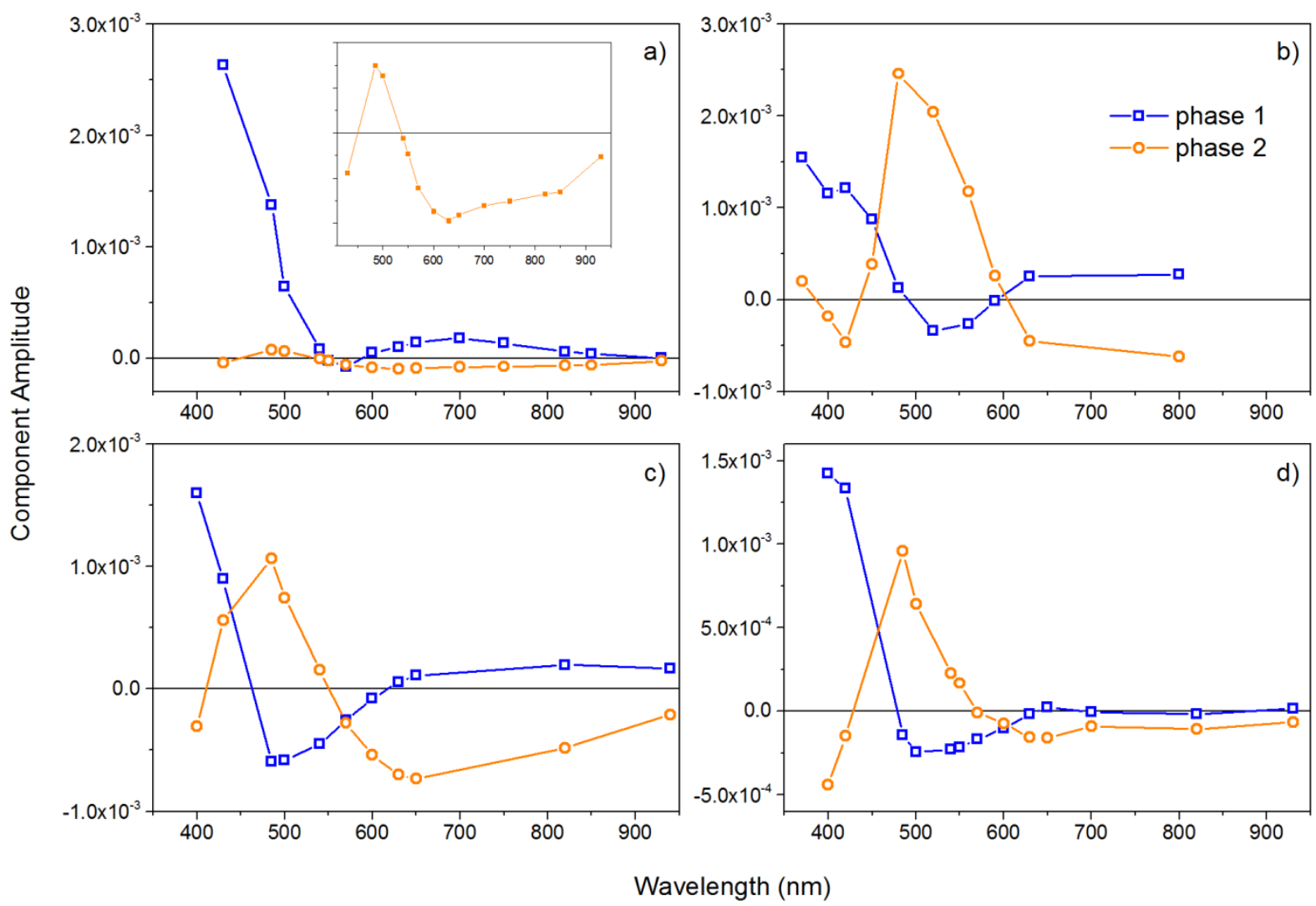

Figure 7. Fitted amplitude parameters, $A_{s p_{i}}$, plotted as a function of the wavelength for: a) sample S-5, b) sample S-6.5 and c) sample S-10 after 3 months of air exposition. d) sample S-10 after 24 months of air exposition. In the inset of (a) the phase 2 component is magnified to better evidence the shape similarity.

Figure 7 shows the amplitude factors $A_{s p_{i}}$ of the two magnetic contributions plotted as a function of light wavelength for all the three investigated systems. This characterization has been performed 3 months after the synthesis for all the samples (Figures 7a-c) and repeated 24 months after the synthesis for S-10 (Figure 7d).

What immediately stands out from Figure 7 is that both MCD contributions maintain quite similar lineshapes for every sample. This fact is an important hint for supporting the validity of 
the procedure of magnetic loop decomposition, as each component appears as clearly identified by a specific spectral signature which can originate from a specific magnetic material.

The two contributions for S-6.5 and S-10 have comparable intensities, suggesting a heterogeneous composition of both systems; on the contrary, in the smallest sample S-5, which is supposed to have been the most subjected to oxidation, the MCD response is mostly due to a single magnetic component, namely phase 1 . In our opinion, this phenomenological result supports the hypothesis of the presence of two distinct magnetic phases in the samples: phase 1, related to maghemite and phase 2 , related to magnetite.

The validity of this hypothesis is further corroborated by literature data. In fact, while the raw MCD spectra of samples S-6.5 and S-10 present a very complex overlap of MO transitions, the spectra resulting from the single magnetic contributions allow for a much easier identification of the spectral features. According to Ref. ${ }^{28}$ the spectral lineshape obtained for phase 2 can be related to $\mathrm{Fe}_{3} \mathrm{O}_{4}$ On the other hand, the $\mathrm{MCD}$ lineshapes arising from phase 1 can be assigned to maghemite..$^{25,29,30}$

The evolution of the MCD components in S-10 after 24 months of air exposition (Figure 7d) gives evidence of the oxidation process. In fact, in the magnetite-related spectrum (phase 2) the IVCT at $640 \mathrm{~nm}$ shows an intensity decrease, as expected due to the reduction of $\mathrm{Fe}^{2+}$ cations. Apparently, the peak located at $500 \mathrm{~nm}$ does not experience a similar intensity loss, but this is likely due to the appearance of two ISCT transitions involving only $\mathrm{Fe}^{3+}$, located around 430 and $480 \mathrm{~nm}:{ }^{23}$ usually these transitions are very weak compared to the IVCT, but in this case they could be boosted by the increase of the $\mathrm{Fe}^{3+}$ ions concentration. 
Summarizing, the analysis presented so far suggests that the magnetic contribution of phase 1 is related to an oxidized moiety, namely $\gamma-\mathrm{Fe}_{2} \mathrm{O}_{3}$. The other magnetic component, phase 2 , is likely related to the initial $\mathrm{Fe}_{3} \mathrm{O}_{4}$ fraction.

The fact that the Verwey transition is progressively less pronounced with decreasing particle size, as shown in ZFC-FC curves of Figure 2, is an additional indication that, the magnetite fraction in the overall Fe oxide tends to diminish until it is no longer the main constituent. This issue, however, is still rather controversial: indeed, although several authors claimed that on decreasing the NPs diameter the Verwey transition shifts to lower temperature and, below a critical size (about $30 \mathrm{~nm}$ ), it disappears, ${ }^{6}$ they do not take into account the possibility that the observed trend might originate from the progressive oxidation of magnetite to maghemite (which is more favored in smaller NPs). On the other hand, some works reported the signature of the Verwey transition temperature in magnetite grains with size much smaller than $30 \mathrm{~nm} .{ }^{31-35}$ For instance, Markovich and co-workers observed an abrupt increase in the resistance on cooling 5.5 $\mathrm{nm}$ magnetite NPs below $96 \mathrm{~K}$ and attributed it to the Verwey metal-insulator transition. ${ }^{31,32}$

To confirm our interpretation, the same analysis method has been applied to the study of three magnetite-maghemite heterogeneous systems obtained by mixing different ratios of two sets of NPs, each one reasonably considered to be made of a single iron oxide phase. In fact, for these samples the lineshape of the MO response originating from each pure component is expected to remain unchanged, the only variable being the weight of the single MCD responses, which is directly related to the amount of correspondent NPs. Taking into account the previous experimental results and considerations, we used S-5b (after 18 months of air exposition) as pure maghemite sample. For the magnetite standard, we synthesized a new sample, S-46, made up of $\mathrm{Fe}_{3} \mathrm{O}_{4}$ NPs with average size of $46 \pm 13 \mathrm{~nm}$ (Fig.S2b in Supporting Information). Due to the 
large size, oxidation issues could be neglected and the sample has been accounted as a reliable nanosized magnetite standard. As a drawback, this sample shows larger size and shape dispersion. In particular, in the largest NPs of the distribution, the atomic magnetic moments are not expected to coherently rotate under the influence of an external field, and, in general, the whole sample is no longer expected to exhibit a purely superparamagnetic behavior at room temperature. The mixed systems, called MIX-A, MIX-B and MIX-C, were prepared by combining and casting in the polymer matrix liquid dispersions of S-5b and S-46 with different concentration ratio. The final calculated ratio between maghemite and magnetite concentrations were, respectively, 0.6:1 for MIX-A, 1.2:1 for MIX-B and 2.5:1 for MIX-C.

In Figure 8 the MCD spectra of S-5b and S-46 are reported together with those of the three MIX. It appears that the MCD spectra of the MIX are quite different from one another, although each one originates from the simple combination of the same components.

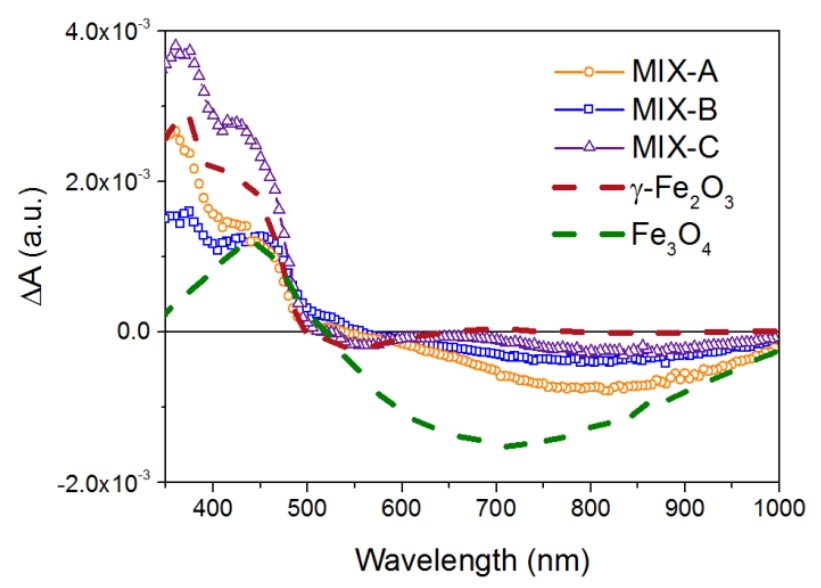

Figure 8. MCD spectra of the standard samples S-46 and S-5b and their combinations MIX-A, MIX-B and MIX-C. The standard spectra have been scaled by $50 \%$ for clarity of presentation. 
First, we applied the procedure described above to the analysis of standard samples. The obtained results confirm that both samples are prevalently composed by a single magnetic phase (Fig. S3, S.I.). Then, the $C_{1}$ and $C_{2}$ parameters were extracted from MCD loops performed on MIX-A, MIX-B and MIX-C. It is worth to stress that these values are identical to those evaluated for the standard samples. The deconvolutions of the MCD spectra for the MIX obtained from the fitting procedure are shown in Figure 9.
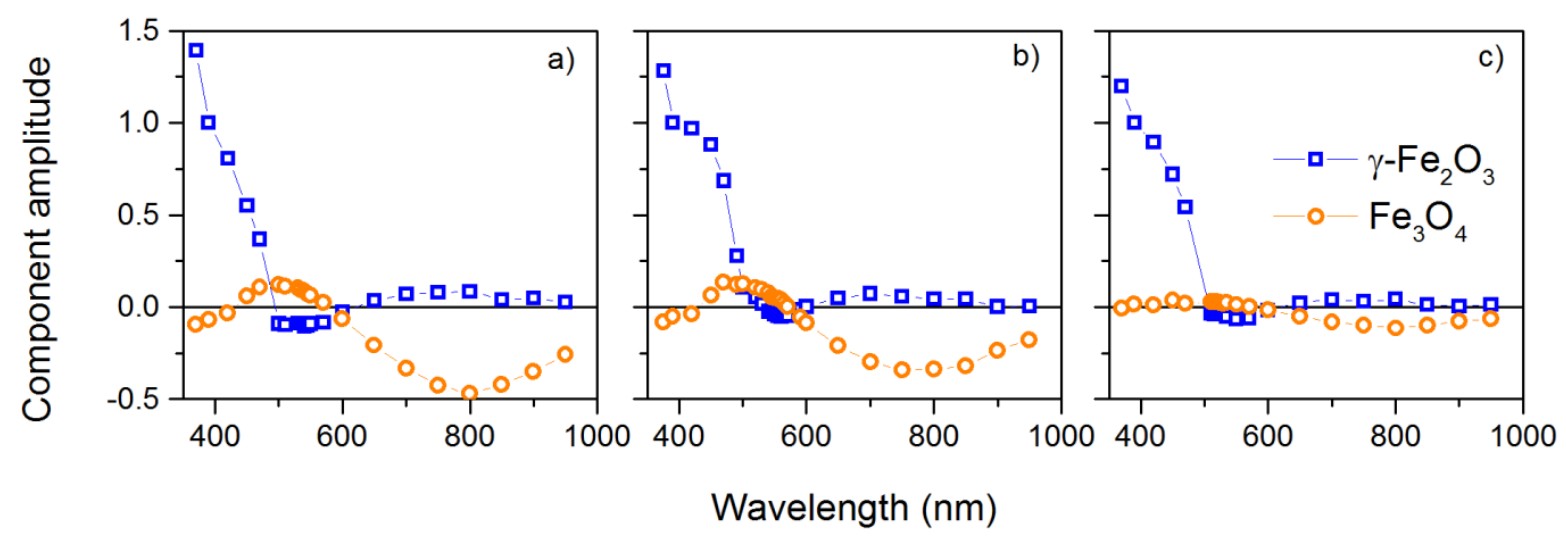

Figure 9. $A_{s p_{i}}$ values plotted as a function of the wavelength obtained as best fit parameters for the maghemite (S-5b) and magnetite (S-46) components in MIX-A (a) MIX-B (b) and MIX-C (c).

For each sample the spectral lineshapes of the two components separated by the deconvolution procedure are essentially identical, except for the relative magnitude of the signal, which is consistent with the variation trend of the relative compositions. In addition, they are very similar to the lineshape of the standard samples and to the components found in the initial series of sample (S-5, S-6.5, S-10), confirming their nature of partially oxidized $\mathrm{Fe}_{3} \mathrm{O}_{4} / \gamma-\mathrm{Fe}_{2} \mathrm{O}_{3}$ 
nanostructures. All these results point out the validity of the proposed approach as an effective tool for the estimation of the oxidation degree in cubic spinel iron oxide.

Our analysis clearly demonstrated that from the experimental MCD spectra-it is already possible to extract from the experimental MCD spectra independently the contributions of two independent magnetic phases and to get qualitatively indications about their relative weight, as shown forin the samples MIX-A, MIX-B and MIX-C, artificially produced by simply mixing two sets of standard NPs. Moreover, these results appear very promising for the future perspective of a true-fully quantitative MCD techniquemethod. Nevertheless, this procedure can be suitable to get information also about nominally "homogeneous" systems, such as the samples S-5, S-6.5 and S-10 investigated in this work. Indeed, considering the narrow size distribution of samples S-6.5 and S-10, it appears reasonable to assume that oxidation occurs gradually and homogeneously in all NPs and does not convert some of them into maghemite while leaving the others in the starting magnetite phase.

Within this framework two phases coexist in the same NP, considering the presence of distinct regions with different chemical composition. Such a structure can be represented by a core-shell morphology, ${ }^{8,9}$ formed by the progressive oxidation of the starting magnetite to maghemite due to the interaction with the surrounding environment, whereas the inner core is protected and keeps the original chemical structure.

We wish to remark that the adopted model neglects magnetic interactions between the two phases and spin disorder originating from surface effects. Although these approximations do not affect the general validity of the proposed method, they compromise the numerical value of the Langevin $C$ parameters (reported in Supporting Information, Table S1) which have to be considered just as mathematical tools for identifying the magnetic-component. 
We conclude that the simple method we propose, based on MO measurements and hysteresis deconvolution, open new perspectives for the use of this technique as an alternative and powerful tool to study the composition of iron oxide nanostructures and their time evolution.

\section{CONCLUSIONS}

We investigated the magnetic and MO properties of a set of iron oxide spinel ferrite NPs with different size. The MCD spectra and the complex magnetic dependence of the MCD loops, performed over a long time span after the NPs were synthesized, were analyzed by means of a deconvolution method. Our analysis suggested the presence of more than one magnetic contribution, which can be explained by the progressive oxidation of the iron oxide NPs from magnetite to maghemite. The dependence on size and the time evolution of the relative composition of the samples are in good agreement with such hypothesis.

These results demonstrate the potentiality of the MO spectroscopy as a fruitful technique for the detection and resolution of multiphase nanometric magnetic systems, which can complement and in some cases overcome the limitations of other well-established methods. Indeed, MO spectroscopy allows for detecting selectively also minor phases, whose signature can be masked by stronger ones in bulk-sensitive measurements. In our opinion, this possibility could be particularly welcome in the field of NP characterization, since in this size regime a complete description of the starting material can hardly be obtained by standard techniques, while reactivity, strain and surface effects can significantly alter chemical and physical properties. Further applications of this experimental technique could be considered for different magnetic nanosized systems as an effective method for assessing the presence of different, hard to spot magnetic phases and checking the oxidation and evolution degree of such small structures. 


\section{SUPPORTING INFORMATION}

Supporting Information Available: XRD diffractograms, TEM images and MCD components deconvolutions of large size magnetite (S-46) and small size maghemite NPs (S-5b). List of values fitted for the $\mathrm{C}$ parameters. This information is available free of charge via the Internet at http://pubs.acs.org/.

\section{AUTHOR INFORMATION}

\section{Corresponding authors}

*e-mail: giulio.campo@unifi.it; csangregorio@iccom.cnr.it

\section{Conflict of interest.}

The authors declare no competing financial interest.

\section{ACKNOWLEDGMENT}

We acknowledge the EC for the financial support through FP7-310516 NANOPYME, the Italian MIUR through FIRB projects RBPR05JH2P Rete ItalNanoNet and Futuro in Ricerca 2010 "NanoPlasMag" (RBFR10OA10), Fondazione Cariplo through the project No. 2010-0612 "Chemical synthesis and characterization of magneto-plasmonic nano-heterostructures" and INSTM-Regione Lombardia through project MAG-NANO. 


\section{REFERENCES}

1. Aurich, K.; Glöckl, G.; Nagel, S.; Weitschies, W. Sensors 2009, 9, (6), 4022-4033.

2. Aurich, K.; Nagel, S.; Glöckl, G.; Weitschies, W. Anal. Chem. 2006, 79, (2), 580-586.

3. Brabers, V. A. M.; Buschow, K. H. J., Handbook of Magnetic Materials. Amsterdam, 1995; Vol. 8.

4. Tran, N.; Webster, T. J. J. Mater. Chem. 2010, 20, (40), 8760-8767.

5. Cornell, R. M.; Schwertmann, U., The Iron Oxides: Structure, Properties, Reactions, Occurrences and Uses. Wiley: 2003.

6. Goya, G. F.; Berquo, T. S.; Fonseca, F. C.; Morales, M. P. J. Appl. Phys. 2003, 94, (5), $3520-3528$.

7. Andrés-Vergés, M.; del Puerto Morales, M.; Veintemillas-Verdaguer, S.; Palomares, F. J.; Serna, C. J. Chem. Mater. 2011, 24, (2), 319-324.

8. Santoyo Salazar, J.; Perez, L.; de Abril, O.; Truong Phuoc, L.; Ihiawakrim, D.; Vazquez, M.; Greneche, J.-M.; Begin-Colin, S.; Pourroy, G. Chem. Mater. 2011, 23, (6), 1379-1386.

9. Frison, R.; Cernuto, G.; Cervellino, A.; Zaharko, O.; Colonna, G. M.; Guagliardi, A.; Masciocchi, N. Chem. Mater. 2013, 25, (23), 4820-4827.

10. De Matteis, L.; Custardoy, L.; Fernández-Pacheco, R.; Magén, C.; de la Fuente, J. M.; Marquina, C.; Ibarra, M. R. Chem. Mater. 2012, 24, (3), 451-456.

11. Singh, N.; Jenkins, G. J.; Asadi, R.; Doak, S. H. Nano Rev. 2010, 1.

12. Walz, F. J. Phys.: Condens. Matter 2002, 14, (12), R285.

13. Espinosa, A.; Serrano, A.; Llavona, A.; de la Morena, J. J.; Abuin, M.; Figuerola, A.; Pellegrino, T.; Fernández, J.; Garcia-Hernandez, M.; Castro, G. Meas. Sci. Technol. 2012, 23, (1), 015602 . 
14. Jain, P. K.; Xiao, Y.; Walsworth, R.; Cohen, A. E. Nano Lett. 2009, 9, (4), 1644-1650.

15. Fantechi, E.; Campo, G.; Carta, D.; Corrias, A.; de Julián Fernández, C.; Gatteschi, D.; Innocenti, C.; Pineider, F.; Rugi, F.; Sangregorio, C. J. Phys. Chem. C 2012, 116, (14), 82618270.

16. Tirosh, E.; Shemer, G.; Markovich, G. Chem. Mater. 2006, 18, (2), 465-470.

17. Mason, W. R., A Practical Guide to Magnetic Circular Dichroism Spectroscopy. WileyInterscience: New York, 2007.

18. Sun, S.; Zeng, H.; Robinson, D. B.; Raoux, S.; Rice, P. M.; Wang, S. X.; Li, G. J. Am. Chem. Soc. 2003, 126, (1), 273-279.

19. Park, J.; An, K.; Hwang, Y.; Park, J.-G.; Noh, H.-J.; Kim, J.-Y.; Park, J.-H.; Hwang, N.M.; Hyeon, T. Nat. Mater. 2004, 3, (12), 891-895.

20. Mason, W. R. Anal. Chem. 1982, 54, (4), 646-648.

21. Fontijn, W. F. J.; van der Zaag, P. J.; Feiner, L. F.; Metselaar, R.; Devillers, M. A. C. J. Appl. Phys. 1999, 85, (8), 5100-5105.

22. Scott, G. B.; Lacklison, D. E.; Ralph, H. I.; Page, J. L. Phys. Rev. B 1975, 12, (7Physical Review B), 2562-2571.

23. Fontijn, W. F. J.; van der Zaag, P. J.; Devillers, M. A. C.; Brabers, V. A. M.; Metselaar, R. Phys. Rev. B 1997, 56, (9), 5432-5442.

24. Kim, K. J.; Lee, H. S.; Lee, M. H.; Lee, S. H. J. Appl. Phys. 2002, 91, (12), 9974-9977.

25. Bentivegna, F.; Nyvlt, M.; Ferre, J.; Jamet, J. P.; Brun, A.; Vis n ovsky, S.; Urban, R. J. Appl. Phys. 1999, 85, (4), 2270-2278.

26. Ferré, J.; Meyer, P.; Nyvlt, M.; Visnovsky, S.; Renard, D. J. Magn. Magn. Mater. 1997, $165,(1-3), 92-95$. 
27. Postava, K.; Sveklo, I.; Tekielak, M.; Mazalski, P.; Maziewski, A.; Stupakiewicz, A.;

Urbaniak, M.; Szymanski, B.; Stobiecki, F. IEEE Trans. Magn. 2008, 44, (11), 3261-3264.

28. Lin, C.; Tseng, Y.; Ovchinnikov, S.; Ivantsov, R.; Edelman, I.; Fedorov, A.; Kuzubov, A.; Fedorov, D.; Starchikov, S.; Lyubutin, I. Mat. Res. Expr. 2014, 1, (2), 025033.

29. He, Y.; Miao, Y.; Li, C.; Wang, S.; Cao, L.; Xie, S.; Yang, G.; Zou, B.; Burda, C. Phys. Rev. B 2005, 71, (12), 125411.

30. Edelman, I.; Ivanova, O.; Ivantsov, R.; Velikanov, D.; Zabluda, V.; Zubavichus, Y.;

Veligzhanin, A.; Zaikovskiy, V.; Stepanov, S.; Artemenko, A.; Curely, J.; Kliava, J. J. Appl.

Phys. 2012, 112, (8), 084331.

31. Poddar, P.; Fried, T.; Markovich, G. Physical Review B 2002, 65, (17), 172405.

32. Markovich, G.; Fried, T.; Poddar, P.; Sharoni, A.; Katz, D.; Wizansky, T.; Millo, O. Mater. Res. Soc. Symp. Proc. 2002, 746.

33. Huang, C.-C.; Tsai, C.-Y.; Sheu, H.-S.; Chuang, K.-Y.; Su, C.-H.; Jeng, U. S.; Cheng, F.Y.; Su, C.-H.; Lei, H.-Y.; Yeh, C.-S. ACS Nano 2011, 5, (5), 3905-3916.

34. Frey, N. A. Magnetics, IEEE Transactions on 2007, 43, (6), 3094 - 3096

35. Muscas, G.; Concas, G.; Cannas, C.; Musinu, A.; Ardu, A.; Orrù, F.; Fiorani, D.; Laureti, S.; Rinaldi, D.; Piccaluga, G.; Peddis, D. J. Phys. Chem. C 2013, 117, (44), 23378-23384. 


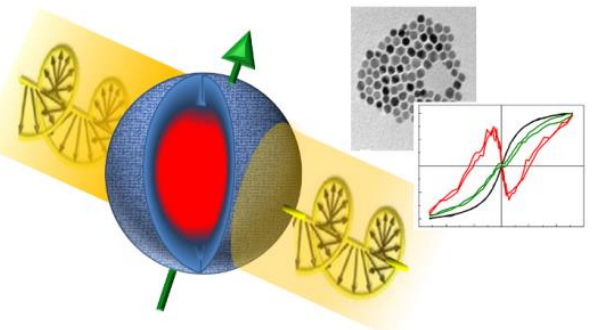

